Marquette University

e-Publications@Marquette

$10-3-2019$

\title{
High-Sensitivity Magnetic Sensors Based on GMI Microwire-SAW IDT Design
}

Akila Khatun

Florian Bender

Fabien Josse PhD

Arnold Kweku Mensah-Brown

R. Dyche Anderson

Follow this and additional works at: https://epublications.marquette.edu/electric_fac

Part of the Computer Engineering Commons, and the Electrical and Computer Engineering Commons 
Marquette University

e-Publications@Marquette

\title{
Electrical and Computer Engineering Faculty Research and Publications/College of Engineering
}

This paper is NOT THE PUBLISHED VERSION; but the author's final, peer-reviewed manuscript. The published version may be accessed by following the link in the citation below.

2019 Joint Conference of the IEEE International Frequency Control Symposium and European Frequency and Time Forum (EFTF/IFC), (October 3, 2019). DOI. This article is (C) Institute of Electrical and Electronic Engineers (IEEE) and permission has been granted for this version to appear in $\underline{\mathrm{e}-}$ Publications@Marquette. Institute of Electrical and Electronic Engineers (IEEE) does not grant permission for this article to be further copied/distributed or hosted elsewhere without the express permission from Institute of Electrical and Electronic Engineers (IEEE).

\section{High-Sensitivity Magnetic Sensors Based on GMI Microwire-SAW IDT Design}

\author{
Akila Khatun \\ Florian Bender \\ Fabien Josse \\ Arnold K. Mensah-Brown \\ Ford Motor Company, Dearborn, Michigan \\ R. Dyche Anderson \\ Ford Motor Company, Dearborn, Michigan
}

Department of Electrical and Computer Engineering, Marquette University, Milwaukee, Wisconsin

Department of Electrical and Computer Engineering, Marquette University, Milwaukee, Wisconsin

Department of Electrical and Computer Engineering, Marquette University, Milwaukee, Wisconsin

\section{Abstract:}

This work presents a design approach for a highly sensitive, miniaturized magnetic sensor. The design makes use of GMI microwires and a multi-electrode SAW IDT. The use of SAW IDTs allows for the magnetic effect of the 
GMI microwire to be measured through the transduction process. This approach permits simultaneous measurement at different frequencies of operation, enabling highly sensitive measurement over a wide range of magnetic fields. This technique may find application in magnetic sensing for non-invasive battery SOC measurement.

\section{SECTION I. Introduction}

Magnetic sensors are used in a variety of fields, including medical, automobile, military, mining, meteorological, archeological and geological applications. Depending on the application, different techniques are used, e.g., Hall effect sensors, magnetoimpedance sensors, fluxgate devices, SQUIDs, etc. In recent decades, a trend towards small magnetic sensors with low power consumption but high sensitivity has been observed. An important step in this development is based on the use of the giant magnetoimpedance (GMI) effect. GMI elements can be fabricated in the shape of microwires, ribbons or thin films and can be combined with surface acoustic wave (SAW) interdigital transducers (IDT), resulting in a magnetic sensor design that has the potential for miniaturization, integration, low cost, passive interrogation, high sensitivity, and large linear range [1].

While GMI microwires enable highly sensitive magnetic field measurement, the dependence of the impedance of GMI wires on magnetic field varies strongly with the frequency of operation [2]. Depending on its composition, the impedance characteristics of a GMI wire will drastically vary, thus limiting the range of magnetic fields where high sensitivity can be achieved [2]. One way to extend highly sensitive magnetic measurement to a larger range of magnetic fields is to operate the GMI wire at different frequencies. Furthermore, the wire has to be stress-free to maximize the GMI effect and thus the sensitivity and reproducibility (hysteresis effect) in magnetic field measurements. This limits the value of the inductance of the wire that can be obtained, although the length of the wire can still be varied. When designed appropriately, the same SAW IDT can be operated at different harmonics, offering a convenient way to design a GMI microwirebased SAW IDT magnetic sensor with high sensitivity in a wide range of magnetic fields. This work presents a magnetic sensor design based on GMI microwires and multi-electrode SAW IDTs to allow sensitive magnetic field measurement at different frequencies.

The eventual field of application of this sensor is the measurement of the change in magnetic susceptibility of lithium ion batteries as a non-invasive way to determine the state of charge (SOC) of the battery [3]. As a first step, this work is characterizing the sensor device to understand the impact of various design factors including GMI-wire length, operating frequency, and sensor configuration that will impact the desired goals of high sensitivity and high resolution.

\section{SECTION II. Experimental Methods}

The GMI wires used in this work consist of amorphous $\mathrm{CO}_{71} \mathrm{Fe}_{4} \mathrm{Si}_{14.5} \mathrm{Nb}_{4} \mathrm{~B}_{6.5}$ fibers of $30 \mu \mathrm{m}$ in diameter [2] and were obtained from MXT, Montreal, Canada. They were connected in parallel to single interdigital transducers (IDTs) deposited on a piezoelectric substrate $\left(36^{\circ} \mathrm{YX}-\mathrm{LiTaO}_{3}\right.$. The multi-electrode SAW IDT design is described in detail in [4]; it has an electrode finger width of $5 \mu \mathrm{m}$, an operating frequency of $34 \mathrm{MHz}$ and harmonics at about 68,104 and $138 \mathrm{MHz}$. The impedance spectra of the resulting GMI wire-IDT device were recorded as a function of magnetic field strength for different SAW harmonic frequencies using a network analyzer (Agilent E5061B, Santa Clara, CA).

\section{SECTION III. Results and Discussion}

In order to understand the sensor response with magnetic field, the equivalent circuit of the GMI microwireSAW IDT combination must be characterized. First, both the impedances of the GMI wire and single SAW IDTs 
were characterized. The IDT equivalent circuit is described in detail in the literature [5]. The impedance of a GMI microwire, $Z_{G M I}$, is given by the equation [6]:

$$
Z_{G M I}=R_{d c} \frac{r}{2 \delta}+j \omega L_{i} \frac{2 \delta}{r}
$$

where, $R_{d c}$ is the DC resistance, $r$ and $\delta$ are the radius and skin depth of the wire, and $L_{i}$ is the internal inductance. Note that $\delta$ depends on both magnetic field strength and frequency, $\omega[6]$.

In agreement with the literature [7], the highest sensitivity to magnetic field was obtained if the GMI wire was oriented parallel to the magnetic field. For this geometry, the sensitivity was measured for GMI microwires of different lengths. Sample results are shown in Fig. 1 for the third SAW harmonic at $104 \mathrm{MHz}$. The highest sensitivity was obtained for a $3 \mathrm{~mm}$ long GMI microwire for lower magnetic field range. It was observed that any deformation or stress will reduce the sensitivity of the GMI microwire, and care was taken to keep the microwire straight and stress free in all following measurements.

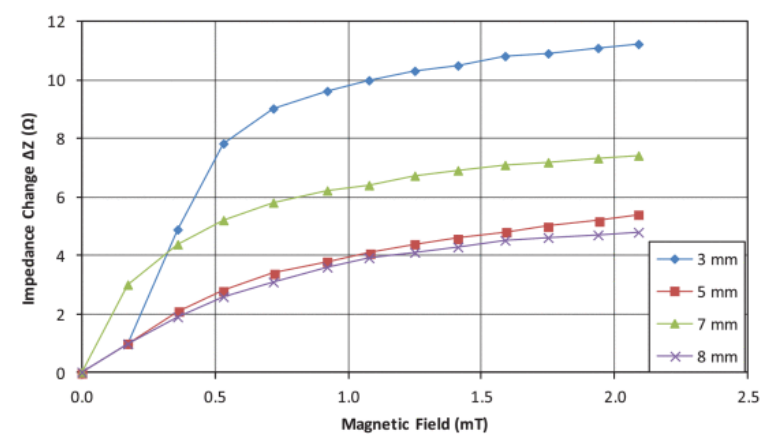

Fig. 1. Change in impedance of magnetic sensor as a function of magnetic field for different lengths of GMI wires as indicated in the graph. Impedance was measured at the third harmonic (104 MHz for this sensor design).

Fig. 2 presents the change in sensor impedance $(Z)$ as a function of magnetic field $(H)$ strength for different SAW frequencies. Results are shown for two different lengths of GMI microwires. In both cases, the sensitivity, $S(S=\partial Z / \partial H)$, for low field values is highest for the 104-MHz mode. This is illustrated in Fig. 2c for the $3 \mathrm{~mm}$ wire. However, for magnetic field values $>0.12 \mathrm{mT}$, the sensor device shows higher sensitivity for the 68- $\mathrm{MHz}$ mode. As a result, simultaneous measurements at different frequencies can be used to achieve high sensitivity over a wide magnetic field range. It is noted that the above observed sensor characteristics can be optimized with different GMI microwires and/or SAW IDTs designs and configurations.

\section{SECTION IV. Conclusions}

It has been shown that, using GMI microwires and multi-frequency SAW IDTs, a magnetic sensor can be designed with high sensitivity over a wider range of magnetic fields. Work is ongoing on the use of this multifrequency approach to compensate for the effect of temperature on the sensor device. This will be necessary for the design of magnetic sensors with high sensitivity and high resolution for measuring the degree of lithiation for lithium ion batteries in environments where temperature changes are encountered. This includes applications involving hybrid electric vehicles (HEVs), plug-in HEV (PHEVs), and battery electric vehicles (BEVs). 

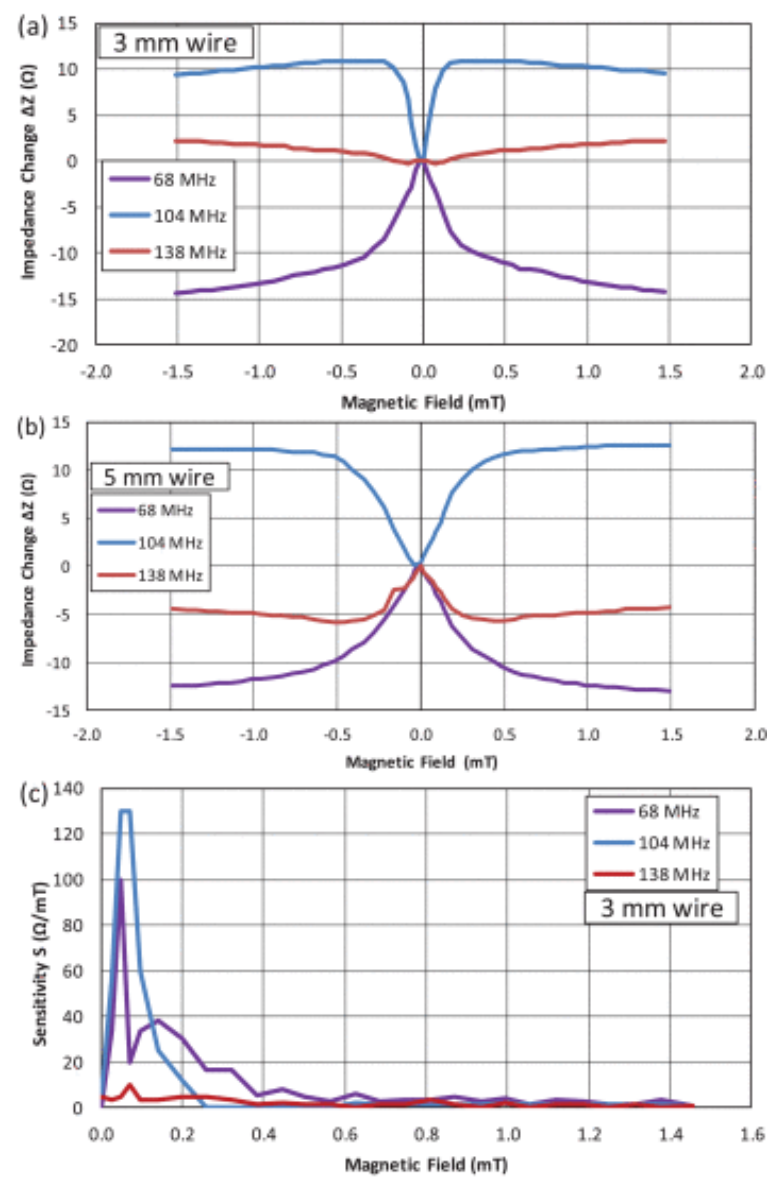

Fig. 2. Change in impedance of magnetic sensors as a function of magnetic field for different SAW frequencies as indicated in the graph. Results are shown for two different GMI wire lengths: (a) $3 \mathrm{~mm}$, (b) $5 \mathrm{~mm}$. (c) Sensitivity, $S(S=\partial \mathrm{Z} / \partial \mathrm{H})$,, for $3 \mathrm{~mm}$ wire length (only shown for positive magnetic field).

\section{References}

1. B. Li, H. Al Rowais, J. Kosel, Surface Acoustic Wave Based Magnetic Sensors, IntechOpen, pp. 355-380, 2013.

2. D. Ménard et al., "High frequency impedance spectra of soft amorphous fibers", J. Appl. Phys., vol. 81, pp. 4032-4034, 1997.

3. T. Kadyk, M. Eikerling, " Magnetic susceptibility as a direct measure of oxidation state in LiFePO 4 batteries and cyclic water gas shift reactors ", Phys. Chem. Chem. Phys., vol. 17, pp. 19834-19843, 2015.

4. F. Bender, R. E. Mohler, A. J. Ricco, F. Josse, "Analysis of Binary Mixtures of Aqueous Aromatic Hydrocarbons with Low-Phase-Noise Shear-Horizontal Surface Acoustic Wave Sensors Using Multielectrode Transducer Designs", Anal. Chem., vol. 86, pp. 11464-11471, 2014.

5. H. Matthews, Surface Wave Filters: Design Construction and Use, New York:Wiley, 1977.

6. H. Hauser, R. Steindl, Ch. Hausleitner, J. Nicolics, A. Pohl, "Miniaturized Magnetic Field Sensors Utilizing Giant Magneto- Impedance [GMI] Effect and Surface Acoustic Wave [SAW] Technology", Sens. Mater., vol. 13, pp. 025-040, 2001.

7. P. Ciureano, G. Rudkowska, P. Rudkowski, J. O. Ström-Olsen, "Magnetoresistive Sensors with Rapidly Solidified Permalloy Fibers", IEEE Trans. Magn., vol. 29, pp. 2251-2257, 1993. 